COMMENT. These attempts to subtype dysphasic children are helpful in the definition of pathogenesis and brain localization and they allow the development of specific intervention strategies. The neurologic bases of developmental dysphasias are probably multiple. Same syndromes may result from genetic abnormalities in brain development while early focal or multifocal acquired brain pathology may be responsible for others. The differentiation of developmental and acquired aphasias in young children may be difficult and subtle cerebral abnormalities may be uncovered by the MRI in children who have otherwise normal neurologic findings. The authors of this article hypothesize that in the course of providing sound therapeutic intervention at an early age they may be able to enhance brain reorganization through the development of altermative pathways. For example, providing dysphasic children of subtype verbal auditory agnosia with a visual language system may promote a bypass of areas of auditory dysfunction and enable the children to acquire verbal language that would have been inaccessible through the auditory channel alone. Failure to show improvement after intensive remediation may be explained by bilateral brain abnormalities that prohibit reorganization. Aut istic children who exhibit severe social attentional and behavioral deficits have the most guarded prognosis.

\title{
PROGNOSIS OF EXPRESSIVE LANGUAGE DELAY
}

The rate of improvement and it's predictive factors have been studied in 26 two year old children with expressive language disorder at the Departments of Pediatrics and Psychology, State University of New York at Stony Brook, and Department of Child Development and Family Relations, University of North Carolina-Greensboro. After a five month follow-up improvement in expressive language was variable, with approximately $1 / 3$ of the children showing no improvement, $1 / 3$ with mild improvement, and $1 / 3$ in the normal range. Three variables measured by the pretest evaluation could be used to predict improvement within the five month period: 1) The size of the child's vocabulary with a cutoff score of $8 ; 2$ ) whether the child was reported as eating regular meals; and 3 ) the extent that the child engaged in quiet "other" behaviors during the mother-child interaction. The more vocabulary the child had used at hame, the more improvement in expressive language was seen. If the child was characterized as not eating three regular meals a day, the less improvement in expressive language was seen. Children who engaged in more periods of quiet noninteractive activity during play and cleanup situations were more likely to improve in expressive language. The reported vocabulary size was $81 \%$ accurate in identifying children's improvement and the prediction of improvement in growth of language. (Fischel, JE et al. Language growth in children with expressive language delay. Pediatrics February 1989; 83:218-227).

COMENT. Expressive language delay is not self-correcting in a short term period of five months for many two year old children. A pediatrician or other professional who routinely advises parents of two year old children with expressive language delay to wait for spontaneous improvement would be wrong $39 \%$ of the time. However, 
these authors found that improvement in expressive skill was not related to a child's involvement in intervention services and the results were not encouraging with regard to the effectiveness of early childhood intervention programs. These findings agree wi th other reports that early intervention efforts did not reduce the frequency of later problems during the school years. The lack of validation of many early intervention efforts noted in the literature places the concerned pediatrician or other professional in a quandary. Apparently, he can use the parent's report of the child's vocabulary size to advise the parent with a high degree of accuracy as to whether the child's expressive language skills will improve in the next few months. However, if the parent wants advice about seeking treatment the basis of action is not clear. The parents should probably be advised of the importance of careful assessment and differential diagnosis in any child with a severe expressive delay and intervention should certainly be considered if the problem persists beyond the third birthday, even given the uncertain effects of intervention techniques. The more accurate diagnosis of subtypes of language delay or dysphasia might lead to more specific intervention techniques as described in the previous article and the beneficial effects of treatment may became more apparent. The early language milestones scale (EIM) has been found a reliable tool to base referral of a child showing evidence of language delay when the test is applied between 25-36 months of age. In the 12 month and younger age groups the test is of no value, and for infants between 13 and 24 months of age there is moderately good agreement between the EIM and the more direct testing with a sequenced inventory of communication development (SICD). (Walker D et al. Early language milestone scale and language screening of young children. Pediatrics Feb 1989; $83: 284-288$ ).

\section{SPEECH ABNOFMALITIES AND TTMPORAL LOBE SEIZURES}

The role of speech manifestations in the lateralization of temporal lobe seizures was reviewed at the Section of Epilepsy and Clinical Neurophysiology, Department of Neurology, Cleveland Clinic Foundation, Cleveland, $\mathrm{OH}$ by the review of videotapes of 100 complex seizures in 35 patients who underwent temporal lobectomy for intractable epilepsy. All patients had prolonged EEG video monitoring with scalp and subdural electrodes and their speech daminance was determined with an intracarotid amobarbital test. Speech manifestations were classified as vocalization, normal speech, or abnormal speech. Vocalizations of sound without speech quality occurred during the seizure in $48.5 \%$ of patients. Normal speech occurred ictally in $34^{\circ}$ of patients. Abnormal speech (speech arrest, dysphasia, and dysarthria) occurred in $51 \%$ of patients either during the seizure or postictally. Only postictal dysphasia and ictal identifiable speech had significant lateralizing value: $92 \%$ of patients with postictal dysphasia had their seizures originating from the daminant temporal lobe, and $83 \%$ of those with ictal identifiable speech had seizures localized to the nondaminant side. (Gabr $M$ et al. Speech manifestations in lateralization of temporal lobe seizures. Ann Neurol Jan 1989; 25:82-87). 